\title{
Strates
}

STRATES Matériaux pour la recherche en sciences sociales

Hors-série | 2002

Parcours dans la recherche urbaine, Michel Rochefort, un géographe engagé

\section{Une civilité métropolitaine engagée}

François Ascher

\section{(2) OpenEdition}

Journals

Édition électronique

URL : http://journals.openedition.org/strates/513

DOI : $10.4000 /$ strates.513

ISSN : 1777-5442

Éditeur

Laboratoire Ladyss

Édition imprimée

Date de publication : 1 janvier 2002

ISSN : 0768-8067

Référence électronique

François Ascher, «Une civilité métropolitaine engagée », Strates [En ligne], Hors-série | 2002, mis en ligne le 02 mai 2005, consulté le 08 septembre 2020. URL : http://journals.openedition.org/strates/ 513 ; DOI : https://doi.org/10.4000/strates.513

Ce document a été généré automatiquement le 8 septembre 2020

Tous droits réservés 


\title{
Une civilité métropolitaine engagée
}

\author{
François Ascher
}

1 La première fois que j'ai rencontré Michel Rochefort, il était incontournable en compagnie de Hautreux et Lecourt dans une référence bibliographique: Le niveau supérieur de l'armature urbaine (Commissariat général au plan, 1963). C'était en 1968-69, j'étais étudiant et je voulais faire de l'économie urbaine.

2 Mais personne ne l'enseignait alors en diplôme d'études supérieures (DES) à la faculté de droit et de sciences économiques de Paris. Il y avait bien un séminaire de Jacques Boudeville à l'Insea, mais on y traduisait surtout des textes nord-américains. Henri Guitton m'avait donc très gentiment accueilli dans son cours, à condition que je fasse un lien entre économie urbaine et conjoncture. Aussi avais-je choisi comme mémoire le sujet suivant : «Conjoncture régionale et économie urbaine : un essai d'application à la Lorraine du Nord». Nous étions alors en pleine période des métropoles d'équilibre. L'Oream s'efforçait de rapprocher et de donner une cohérence globale à un ensemble d'agglomérations qui s'étendaient du nord au sud sur 120 kilomètres, de Longwy à Lunéville en passant par Briey et Thionville, Metz, Pont-à-Mousson et Nancy.

3 Nous avions développé avec Marie-Ève Gagey avec qui je faisais ce mémoire trois thèses principales: la première était que le développement et les politiques économiques devaient s'appuyer beaucoup plus sur une approche spatiale, ce qui était un plaidoyer pour l'économie urbaine et la prise en compte par les économistes de l'espace; la seconde était que le dépassement de la crise de la Lorraine, due apparemment aux difficultés de la sidérurgie, passait bien fondamentalement par l'amélioration de l'armature urbaine et le développement d'une économie diversifiée et de services de niveau supérieur; la troisième thèse enfin, qui était bien sûr influencée par mes origines messines, était que la Lorraine ne formait pas un ensemble cohérent, que le projet de métropole d'équilibre était trop étendu, qu'en revanche la partie nord de la Lorraine avait une cohérence potentielle et qu'il fallait donc ne pas mélanger les projets pour Metz et Nancy.

4 Probablement, si j'avais aujourd'hui à me repencher sur ce type de question, aurai-je des conclusions sensiblement différentes, quitte à considérer que cette vaste conurbation distendue, fragmentée, hétérogène, préfigurait d'une certaine manière les 
espaces «métropolitains" contemporains. Pour autant, je ne crois pas que toute la problématique en termes d'armature urbaine soit complètement obsolète et la notion de services supérieurs me semble même plus que jamais d'actualité. Il faut bien sûr se débarrasser de cette notion d'équilibre qui n'a pas beaucoup de sens. Mais il ne faut pas tout rejeter et il importe au contraire de souligner combien la notion actuelle et très féconde de "métropolisation " doit aux travaux des années soixante et en particulier aux contributions de Michel Rochefort. Mais celui-ci est trop modeste et l'une des critiques majeures que l'on peut faire à un de ses ouvrages récents ${ }^{1}$, qui constitue par ailleurs une excellente synthèse des réflexions actuelles, c'est qu'il ne cite même pas Hautreux et Rochefort, La fonction régionale dans l'armature urbaine française (1964), ouvrage qui a servi de référence pendant des années aux politiques d'aménagement du territoire, et qui a été un précurseur des approches urbaines actuelles.

La seconde fois que j'ai rencontré Michel Rochefort, c'était au Conseil national des universités. À cette époque (déjà ?), c'était un lieu redoutable. En tout cas pour moi qui y siégais depuis des années, c'était un lieu particulièrement hostile : j'y étais triplement marginal, comme élu syndicaliste de gauche, comme non-géographe (il n'y avait plus de section d'urbanisme aux CSU et CNU depuis de nombreuses années) et comme maîtreassistant. Michel Rochefort est alors arrivé. Et tout a changé quand il a été élu président. Pourtant, présider un tel comité dans le contexte d'alors n'était pas facile, d'autant que les oppositions avaient plutôt tendance à s'exacerber. Mais c'est précisément dans ces circonstances que Michel Rochefort est le plus efficace, tout en donnant l'impression que les choses sont simples. Pour dénouer les conflits, Michel s'appuie d'abord sur sa capacité d'écoute. Ensuite, sa propre civilité, dont je ne l'ai pas vu se départir, impose à tous le respect de règles de politesse dont ces assemblées ne sont pas toujours respectueuses. Enfin, il est vraiment très habile, car il sait élaborer des propositions qui ont toutes les allures de compromis, mais qui en fait n'en sont pas! Car Michel Rochefort prend des positions, mais elles tiennent compte autant qu'il est possible des désaccords et des divergences. Avec lui, le CNU a gagné en respectabilité. Plus tard, il n'a pas hésité à soutenir les enseignants des instituts d'urbanisme qui réclamaient la reconstitution d'une section autonome, distincte des géographes qui avaient essayé de contrôler ce nouveau champ universitaire. Car Michel Rochefort, tout en restant géographe, avait pris la mesure de la multidisciplinarité de ce domaine, qui n'est certes pas une nouvelle discipline scientifique, mais qui constitue un vaste secteur de pratiques professionnelles et un enjeu de formation pour les universités.

6 Ma troisième expérience de travail avec Michel Rochefort, date de l'époque où j'étais directeur de ce qui allait devenir l'Institut français d'urbanisme ; il avait accepté alors de présider notre conseil d'administration. C'était une période institutionnelle assez difficile et les relations que nous avions avec notre université comme avec le ministère n'étaient pas toujours bonnes. Michel a su nous consacrer du temps, nous écouter, comprendre et nous aider à résoudre la plupart des conflits qui risquaient d'hypothéquer l'avenir de notre institut. Sa connaissance de l'université et du ministère de l'Éducation nationale, des textes réglementaires comme des coutumes, ont été d'une redoutable efficacité. À titre personnel, je dois dire aussi que Michel, non seulement m'a donné de bons conseils, mais m'a aidé à mieux solliciter et entendre les opinions des uns et des autres.

7 Ma quatrième expérience de travail avec Rochefort fut la rédaction d'un ouvrage collectif auquel participaient également Lucien Brams, Aliette Delamarre et Serge 
Wachter. La Datar nous avait demandé de faire une synthèse et des scénarios prospectifs à partir des résultats des travaux des différents groupes de recherche prospective lancés par Jean-Louis Guigou, directeur, et Jean-Pierre Duport, délégué. Cette expérience fut vraiment enrichissante et la présence de Michel Rochefort fut encore une fois décisive car il réunissait des compétences particulières sur la question des métropoles, en cartographie... et en dynamique de groupe. De fait, je me suis rendu compte à cette occasion qu'il se passe souvent une chose assez curieuse lorsque l'on sort d'une réunion avec Michel : généralement, on lui dit merci.

À ces diverses expériences de travail s'ajoutent évidemment de multiples autres occasions de rencontre avec Michel Rochefort, dans des colloques, des conférences, des comités, des jurys, et depuis un an à nouveau au conseil d'administration de l'IFU, qu'il préside et où son intervention a été décisive pour sauver une institution qui était véritablement en danger. Michel est peut-être un retraité de l'enseignement. Mais il est loin d'être à la retraite, car il profite de son nouveau statut pour rendre encore des services éminents qu'il est d'ailleurs le seul à pouvoir rendre.

9 Les quelques lignes qui précèdent témoignent trop brièvement et incomplètement de l'estime que j'ai pour Michel Rochefort et de toute la reconnaissance que je lui dois. Mais je voudrais profiter aussi de l'hommage que nous lui rendons ici pour lui témoigner de l'affection que je lui porte, et pour lui dire combien pour moi il personnifie les valeurs universitaires, politiques et humaines qui me servent de référence.

\section{NOTES}

1.Michel Rochefort, Dynamique de l'espace français et aménagement du territoire, Éditions L'Harmattan, Paris, 1995, 138 p.

\section{AUTEUR}

\section{FRANÇOIS ASCHER}

Professeur à l'Institut français d'urbanisme à l'université de Paris 8-Saint Denis 